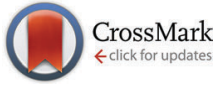

Cite this: J. Mater. Chem. C, 2017, 5,452

Received 14th November 2016, Accepted 15th December 2016

DOI: $10.1039 / \mathrm{c} 6 \mathrm{tc} 04964 \mathrm{~h}$

www.rsc.org/MaterialsC

\title{
Measurement and modelling of dark current decay transients in perovskite solar cells $\$$
}

\author{
Simon E. J. O'Kane, $\S^{\mathrm{a}}$ Giles Richardson, $\S^{\mathrm{b}}$ Adam Pockett, ${ }^{\mathrm{c}}$ Ralf G. Niemann, ${ }^{\mathrm{c}}$ \\ James M. Cave, ${ }^{* a}$ Nobuya Sakai, ${ }^{d}$ Giles E. Eperon, ${ }^{d}$ Henry J. Snaith, ${ }^{d}$ \\ Jamie M. Foster, ${ }^{e}$ Petra J. Cameron ${ }^{c}$ and Alison B. Walker ${ }^{a}$
}

\begin{abstract}
The current decay in response to a sudden change of applied bias up to $1 \mathrm{~V}$ has been measured on a methylammonium lead triiodide perovskite solar cell with titania and spiro-OMeTAD transport layers, for temperatures between 258 and $308 \mathrm{~K}$. These measurements are highly reproducible, in contrast to most other techniques used to investigate perovskite cells. A drift-diffusion model that accounts for slow moving ions as well as electrons and holes acting as charge carriers was used to predict the current transients. The close fit of the model predictions to the measurements shows that mobile ions in the perovskite layer influence transient behaviour on timescales of up to $50 \mathrm{~s}$. An activation energy of $0.55 \mathrm{eV}$ is inferred from fitting simulations to measurements made at room temperature.
\end{abstract}

\section{Introduction}

Since their invention in $2009,{ }^{1}$ organometal halide perovskite solar cells have reached power conversion efficiencies (PCEs) of over $20 \%$. Recent reviews of perovskite solar cell technologies are given by Sum et al., ${ }^{2}$ Stranks and Snaith, ${ }^{3}$ Niu et al., ${ }^{4}$ Miyasaka $^{5}$ and Park. ${ }^{6}$ Perovskites are the subject of a great deal of research interest due to their potential for easier and lower-cost manufacture than the market-leading silicon-based technologies.

Historically, the credibility of reported PCE values for perovskite solar cells has been undermined by widespread use of a voltage scanning protocol that takes advantage of hysteresis in the current density-voltage $(J-V)$ characteristics of some cells to obtain high initial PCE values that decrease as the cell is held at the maximum power point (MPP) voltage. Zhang et al. ${ }^{7}$ measured how the efficiency fell over time for different cell architectures: in the most extreme case the PCE fell from $11.5 \%$ to zero. Hysteresis in the current-voltage characteristics of perovskite solar cells was first reported in $2014^{8}$ and confirmed by subsequent studies, ${ }^{9-12}$ although hysteresis loops in conductance measurements of bulk perovskite had been reported earlier. $^{13}$

\footnotetext{
${ }^{a}$ Department of Physics, University of Bath, UK. E-mail: j.m.cave@bath.ac.uk

${ }^{b}$ Mathematical Sciences, University of Southampton, UK

${ }^{c}$ Department of Chemistry, University of Bath, UK

${ }^{d}$ Department of Physics, University of Oxford, UK

${ }^{e}$ Department of Mathematics, University of Portsmouth, UK

$\dagger$ The data used to produce Fig. $4-6$ and 8 and ESI Fig. S1 are available at DOI: 10.15125/BATH-00251.

\$ Electronic supplementary information (ESI) available. See DOI: 10.1039/c6tc04964h $\S$ Authors contributed equally to this work.
}

In most perovskite solar cells, the organometal halide perovskite absorber is sandwiched between an electron transporting (hole blocking) layer and a hole transporting (electron blocking) layer. ${ }^{14}$ One of the most commonly used, ${ }^{15}$ and indeed longest used, ${ }^{1}$ perovskites is methylammonium lead iodide $\left(\mathrm{CH}_{3} \mathrm{NH}_{3} \mathrm{PbI}_{3}\right)$, or MAPI. The methylammonium $\left(\mathrm{CH}_{3} \mathrm{NH}_{3}{ }^{+}\right)$cation has been successfully replaced with formamidinium ${ }^{16}\left(\mathrm{NH}_{2} \mathrm{CHNH}_{2}{ }^{+}\right)$and/or caesium $^{17}\left(\mathrm{Cs}^{+}\right)$; a mixture of these cations can also be used. ${ }^{18,19}$ There are efforts to replace lead $\left(\mathrm{Pb}^{2+}\right)$ with tin $\left(\mathrm{Sn}^{2+}\right)$, though this usually comes at the expense of performance and stability. ${ }^{20}$ Iodide can be replaced with other halides, either entirely or in a mixture. ${ }^{3,21,22}$ The highest efficiency perovskite cells employ mixed-cation lead mixed-halides. ${ }^{18,23-25}$

The hole transporting layer (HTL) usually consists of spiroOMeTAD (2,2',7,7'-tetrakis-( $N, N$-di- $p$-methoxyphenylamine)-9,9'spirobifluorene) that forms a planar heterojunction with the perovskite. The most commonly used electron transporting layer (ETL) is titania $\left(\mathrm{TiO}_{2}\right)$, which can form either a mesostructured ${ }^{1}$ or planar $^{26}$ heterojunction with the perovskite.

Recently, there have been a number of well-documented 'hysteresis-free' cells ${ }^{27-31}$ in which the titania ETL is replaced with a different material, popularly the fullerene derivative PCBM (phenyl- $\mathrm{C}_{61}$-butyric acid methyl ester). Often the spiro HTL is replaced as well, such as with the polymer PEDOT:PSS (poly(3,4-ethylenedioxythiophene)polystyrene sulfonate). It has been argued that the removal of hysteresis by this change in the blocking materials demonstrates that ion motion in the perovskite is not the source of hysteresis. ${ }^{27,32}$ However it is notable that Bryant et $a{ }^{33}{ }^{33}$ demonstrate that apparently hysteresis-free cells employing PEDOT:PSS, MAPI and PCBM still exhibit significant hysteresis when cooled to $175 \mathrm{~K}$ and that hysteresis 
under normal operating conditions depends on the choice of transport layer. ${ }^{34}$

In addition to hysteresis in both conductance and currentvoltage measurements, unusual features have been observed in impedance spectroscopy, ${ }^{8,35,36}$ intensity-modulated photocurrent spectroscopy (IMPS) ${ }^{35,36}$ and open-circuit voltage decay. ${ }^{36-38}$ These anomalies occur on the same timescale as the hysteresis, suggesting that they may have a common origin.

It was originally suspected ${ }^{13,39}$ that hysteresis was caused by the creation of ferroelectric domains via dipoles in the methylammonium $\left(\mathrm{CH}_{3} \mathrm{NH}_{3}{ }^{+}\right)$ions, but this hypothesis has now been ruled out by electrical measurements. ${ }^{40}$ Two other possible origins of hysteresis have been proposed: large-scale trapping of electrons $s^{27,41,42}$ and mobile ions in the perovskite film..$^{40,43,44}$ Yang et al. ${ }^{45}$ identified that iodide $\left(\mathrm{I}^{-}\right)$ions were moving, either via interstitials or vacancies. Walsh et al. ${ }^{46}$ calculated that the activation energy required to form Schottky defects (vacancy pairs) was much lower than that required to form Frenkel defects (an interstitial and a vacancy). Several computational studies ${ }^{47-50}$ have been conducted in order to calculate the activation energies for the migration of each ionic species in MAPI and other perovskites; all agree that $\mathrm{I}^{-}$is the most mobile ion species, although the calculated values of activation energy for $\mathrm{I}^{-}$ions vary between $0.08 \mathrm{eV}$ and $0.58 \mathrm{eV}$.

While activation energies allow calculation of the rate at which ions move, the only way to evaluate the effect of ion motion on current-voltage characteristics is with electrical transport modelling. Similar to ions in an electrolyte, ionic charge accumulates in Debye layers (also termed double layers) at the edge of the perovskite under the influence of the built-in field. ${ }^{51}$ Li et al. ${ }^{52}$ used X-ray photoemission spectroscopy to measure the iodine to lead $(\mathrm{I} / \mathrm{Pb})$ ratio at various points in a MAPI film. They found that application of an external field increased the $\mathrm{I} / \mathrm{Pb}$ ratio at the positive electrode, indicating a build-up of $\mathrm{I}^{-}$ions, and decreased the ratio at the negative electrode, indicating a depletion of $\mathrm{I}^{-}$ ions. Electron and hole densities are negligible in comparison to ion vacancy densities at all applied voltages up to and including the built-in voltage. The result of this build-up of net charge is band bending, as postulated by Li et $_{\text {al. }}{ }^{52}$ and others. ${ }^{12,47,51,53}$ The band structure of the film as seen by charge carriers is modified electrostatically by the Debye layers, which act to screen the built-in field.

The Debye length is a measure of the characteristic width of these layers and is over two orders of magnitude smaller then the perovskite layer width $(\sim 1.5 \mathrm{~nm}$ compared to hundreds of $\mathrm{nm})$ at the high ion vacancy densities encountered in perovskites. These narrow layers create a major computational challenge when computing the solution over the entire width of the perovskite layer as a very fine mesh spacing is required to represent the structure of the Debye layers. Richardson et al. ${ }^{51}$ argued that electron and hole densities are negligible in comparison to ion vacancy densities at all applied voltages up to and including the built-in voltage. They could then use the method of matched asymptotic expansions to avoid this pitfall and model the Debye layers accurately, providing quantitative agreement with experimental current-voltage curves in a model assuming mobile ion vacancies and trap-assisted electron-hole recombination. Van Reenen et al. ${ }^{54}$ also found that both ion motion and electron trapping were required to explain the current-voltage hysteresis of the type observed in the literature but were unable to reproduce results in the physically relevant regime, nor to achieve quantitative comparison with experiment. We suggest a contributory factor is that the resolution of the finite difference method employed by these authors was too coarse $(4 \mathrm{~nm})$ to accurately resolve the narrow Debye layers.

In this article we explore the effects of ion motion in perovskite solar cells by measuring their dark current decay transients. These measurements are made in the dark by causing the applied potential difference to jump from equilibrium, at $0 \mathrm{~V}$, to $V_{\text {jump }}$ and measuring the resulting current flow through the cell as a function of time. Prior to the measurement the cell is held in the dark at open circuit for sufficient time to allow it to equilibrate (up to an hour at low temperatures). No more than $1 \mathrm{mV}$ of residual voltage could remain after each measurement for reproducibility on this level. This procedure ensures reproducibility of the observed current trace by eliminating preconditioning effects; indeed we have shown that repeating the experiment on the same device by allowing it to equilibrate again after a measurement gives a result that exactly overlays the previous one. In contrast, most reported measured current-voltage characteristics suffer from irreproducibility each time the measurement is made the results are different, even for the same device, as a result of residual preconditioning from previous measurements, slight changes in illumination etc.

Obtaining this repeatability allows us to eliminate extraneous effects such as cell degradation and material changes arising from exposure of the perovskite to light, the latter was discussed in Gottesman et al. ${ }^{55}$ The measured current through a cell depends on the ability of charge carriers to traverse the energy landscape of the materials. As we later discuss in detail, ion motion has a significant effect on the electrostatic potential within the perovskite layer. By probing the physics in this relatively simple setup, we gain crucial insight into the operation of perovskite cells during standard lab tests such as $J-V$ curve sweeps and stabilised power conversion efficiency measurements, and how, and to what extent, ion motion may affect the results. We therefore improve our understanding of how perovskite-based cells behave under normal generating conditions, and what material advantages may be exploited and what barriers need to be overcome.

Here, we report measured dark current decay transients and use the asymptotic method of Richardson $e t a l .{ }^{51}$ along with the experimental results to perform a drift-diffusion simulation on the system. We assume the diffusion coefficient $D_{+}(T)$ of iodide ions shows Arrhenius behaviour as a function of temperature. ${ }^{47}$ By measuring and simulating the transients at a fixed temperature for a range of $V_{\text {jump }}$, we deduce a value for the activation energy for iodide ion movement for our cell. We then use the obtained $D_{+}(T)$ to simulate transients for a fixed $V_{\text {jump }}$ at a variety of temperatures and compare to experiment. Experiment and model employ a FTO:TiO 2 :MAPI:spiro-OMeTAD:Au cell, as shown in Fig. 1. The cell was fabricated using the chloride precursor route as described in Section 2.4. 


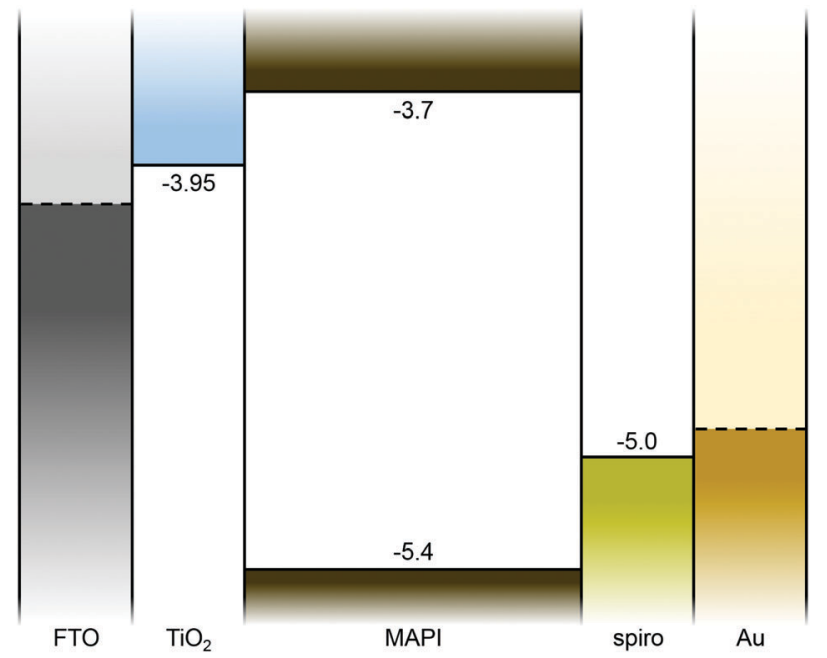

Fig. 1 Band diagram of the device. Band edges for the perovskite and transport layers are represented by solid lines, with energies relative to the vacuum level in eV labelled. ${ }^{56}$ Fermi levels for the contacts are depicted by dotted lines - values for these levels were not required by the model and are difficult to measure accurately, so are not given.

By using the asymptotic model, most of the features observed in the experimental transients can be understood in terms of ion motion. The structure in our transient results (Fig. 5 and 8) provides the first direct evidence that the rate of charge injection into the device, i.e. the current, is dependent on the position of mobile ions within the cell. This result is a corroboration of our earlier work suggesting ion motion is responsible for the widely observed current-voltage hysteresis in perovskite devices. ${ }^{51}$ Analysis of the effect of temperature variation on the timescale of the transients confirms the thermally activated mechanism of ion migration assumed by Eames et al. ${ }^{47}$ and others. ${ }^{48-50}$

\section{Methods}

\subsection{Drift-diffusion model}

The drift-diffusion model used in this work (see Richardson et al. ${ }^{51}$ for details) couples electron and hole charge transport to iodide ion vacancy motion (other vacancies are assumed to be immobile) within a perovskite layer and assumes that the contacts with the blocking layers can be treated as metallic because of the high doping densities in these layers.

We implement this model in a two-step process. Firstly, the electric potential $\phi(x, t)$ - a function of position and time - is found using a time-dependent asymptotic model that considers solely the mobile $\mathrm{I}^{-}$vacancies and their effect on the potential, assuming that the contribution of electrons and holes to the potential is negligible. A schematic diagram of the Debye layer at the MAPI:spiro interface as modelled in this step is illustrated in Fig. 2. We denote the position of the interface of the perovskite with the titania as $x=0$, and the interface of the perovskite with the spiro as $x=b$. As the Debye layers are very thin compared to the perovskite film width, we further denote the edge of the Debye layer on the titania side of the perovskite film as $x=0^{+}$and

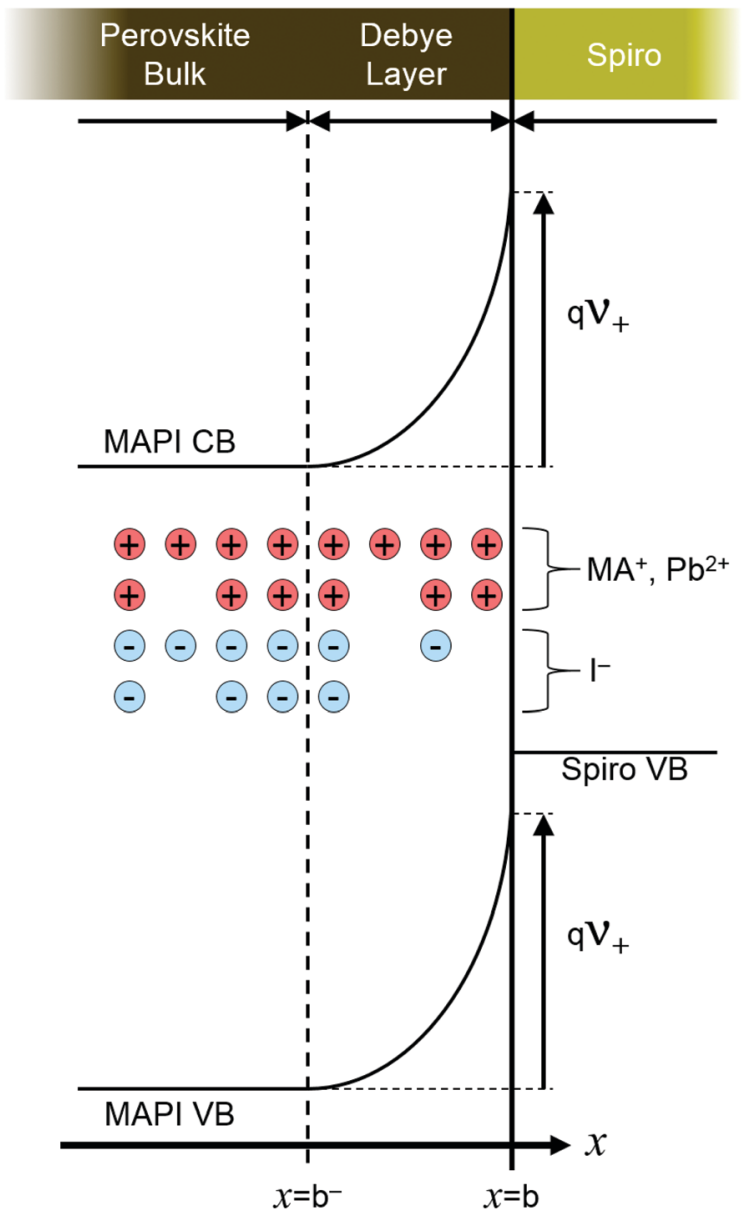

Fig. 2 Schematic of the band structure at the MAPI:spiro interface. Depletion of negative iodide ions (blue circles) from the region adjacent to the interface forms a positively-charged Debye layer. This results in a potential change $\mathcal{V}_{+}$across the layer.

the edge of the layer on the spiro side as $x=b^{-}$. The distinction between $b$ and $b^{-}$is illustrated in Fig. 2. Secondly, a driftdiffusion calculation of electron and hole motion is performed using this potential profile which can then be used to determine the output current density $J(t)$. An accurate calculation of the potential $\phi(x, t)$ and $\mathrm{I}^{-}$vacancy motion can be made by using an asymptotic method ${ }^{51}$ (based on earlier work ${ }^{57}$ ) that overcomes the numerical difficulties encountered when tackling this stiff problem and accounts for the vacancy charge build-up in the narrow Debye layers (of size comparable to the Debye length $L_{\mathrm{d}}$ ) in terms of a nonlinear capacitance.

The model and its asymptotic solution have previously been described in detail ${ }^{51}$ and are briefly summarized here. For volume-averaged vacancy concentrations $N_{0}>10^{17} \mathrm{~cm}^{-3}$ it can be assumed that the main contributions to space charge in the perovskite are concentrated in the Debye layers adjacent to the $\mathrm{TiO}_{2}$ and spiro blocking layers. The bulk of the perovskite between these two layers is therefore free of net charge.

Assuming that only the positively charged $\mathrm{I}^{-}$vacancies can move and the negatively charged $\mathrm{CH}_{3} \mathrm{NH}_{3}{ }^{+}$and $\mathrm{Pb}^{2+}$ vacancies are static, the charge per unit area $Q$ in these layers is related to 
the voltage drop $V$ across them by the asymmetric capacitance relation

$$
Q(\mathcal{V})=\frac{\varepsilon_{\mathrm{p}} V_{\mathrm{T}}}{L_{\mathrm{d}}} \operatorname{sign}(\mathcal{V}) \sqrt{2\left(\exp \left(\frac{\mathcal{V}}{V_{\mathrm{T}}}\right)-1-\frac{\mathcal{V}}{V_{\mathrm{T}}}\right)},
$$

where $\varepsilon_{\mathrm{p}}$ is the permittivity of the perovskite and $V_{\mathrm{T}}$ is the thermal voltage. We denote the surface charge density in the Debye layers adjacent to the spiro and $\mathrm{TiO}_{2}$ as $Q_{+}$and $Q_{-}$ respectively, and the magnitude of the potential drops across these layers by $\mathcal{V}_{+}$and $-\mathcal{V}_{-}$respectively. Due to the large donor and acceptor densities in the $\mathrm{TiO}_{2}$ and spiro, ${ }^{6,58}$ leading to large effective relative permittivities, the potential drop across these blocking layers is, to a good approximation, zero. The total potential drop across the perovskite layer is thus approximately equal to that across the entire cell, $V_{\mathrm{bi}}-V$, where $V_{\mathrm{bi}}$ is the built-in voltage and $V$ the externally applied voltage. The total potential drop across the charge-neutral bulk perovskite region (between the Debye layers) is simply the total drop across the perovskite less the drops across the Debye layers. As the widths of the Debye layers are small compared to the width of the entire perovskite layer $(b=450 \mathrm{~nm})$, the electric field $E_{\text {bulk }}$ across the bulk region is given by

$$
E_{\text {bulk }} \approx \frac{\left(V_{\mathrm{bi}}-V\right)-\mathcal{V}_{+}+\mathcal{V}_{-}}{b} \text {. }
$$

The potential is higher at the $\mathrm{TiO}_{2}$ interface than the spiro interface if the applied voltage $V$ is less than the built-in voltage $V_{\text {bi }}$, which without Debye layers results in a positive $E_{\text {bulk. }}$ A positive value of $E_{\text {bulk }}$ results in electrons being driven towards the $\mathrm{TiO}_{2}$ and holes towards the spiro, which is desirable under normal solar operating conditions. The Debye layers act to cancel $E_{\text {bulk }}$, however, reducing charge extraction efficiency.

By considering charge conservation and the charge neutrality of the system, it can be shown ${ }^{51}$ that the problem of determining the electric field can be reduced to determining the solution of the single ordinary differential equation

$$
\frac{\mathrm{d} Q_{+}}{\mathrm{d} t}=\frac{q D_{+} N_{0}}{V_{\mathrm{T}}} E_{\mathrm{bulk}}(t)
$$

where $Q_{+}$is the areal charge density in the Debye layer next to the spiro-OMeTAD hole-transporting layer, $D_{+}$the iodide vacancy diffusion coefficient, $N_{0}$ the average iodide vacancy density and $V_{\mathrm{T}}$ the thermal voltage.

The difference between the model here and that reported in Richardson et $a .^{51}$ is that here we model recombination by a combination of a bimolecular scheme within the perovskite and so-called surface recombination between an electron in the $\mathrm{TiO}_{2}$ conduction band and a hole in the MAPI valence band across the interface. The bimolecular recombination rate $R_{\mathrm{b}}$ is given by

$$
R_{\mathrm{b}}(x, t)=B\left(n p-n_{\mathrm{i}}^{2}\right),
$$

where $p(x, t)$ and $n(x, t)$ are the hole and electron densities, $n_{\mathrm{i}}$ the intrinsic carrier density and $B$ the bimolecular recombination coefficient (a constant). The surface recombination rate between an electron in the titania and a hole at the edge of the Debye layer on the titania side of the perovskite is given by

$$
R_{\mathrm{s}}\left(x=0^{+}, t\right)=v_{\mathrm{s}} p\left(x=0^{+}, t\right)
$$

where $v_{\mathrm{s}}$ is the surface recombination velocity and $p\left(x=0^{+}, t\right)$ is the hole density in the perovskite at the edge of the Debye layer.

\subsection{Choice of parameters}

Table 1 shows the parameter definitions and values used in our model. The (temperature dependent) electron density in the perovskite at the titania interface $n(x=0)$, hole density at the spiro interface $p(x=b)$, intrinsic carrier density $n_{\mathrm{i}}(T)$ and thermal voltage $V_{\mathrm{T}}(T)$ are given by

$$
\begin{aligned}
& n(x=0)=\hat{g}_{\mathrm{c}} \exp \left(\frac{E_{\mathrm{Ft}}-E_{\mathrm{C}}}{k_{\mathrm{B}} T}\right), \\
& p(x=b)=\hat{g}_{\mathrm{v}} \exp \left(\frac{E_{\mathrm{V}}-E_{\mathrm{Fs}}}{k_{\mathrm{B}} T}\right), \\
& n_{\mathrm{i}}(T)=\sqrt{\hat{g}_{\mathrm{c}} \hat{g}_{\mathrm{v}}} \exp \left(-\frac{E_{\mathrm{g}}}{2 k_{\mathrm{B}} T}\right),
\end{aligned}
$$

\begin{tabular}{|c|c|c|c|}
\hline$D_{\infty}$ & High $T$ vacancy diffusion coefficient & $1.35 \times 10^{-3} \mathrm{~cm}^{2} \mathrm{~s}^{-1}$ & Eames et $a l^{47}$ \\
\hline$E_{\mathrm{Fs}}$ & Spiro-OMeTAD Fermi level & $-5.0 \mathrm{eV}$ & Schulz et al. ${ }^{56}$ \\
\hline$E_{\mathrm{Ft}}$ & $\mathrm{TiO}_{2}$ Fermi level & $-3.95 \mathrm{eV}$ & Schulz et al. ${ }^{56}$ \\
\hline$E_{\mathrm{g}}$ & Perovskite bandgap & $1.7 \mathrm{eV}$ & $=E_{\mathrm{C}}-E_{\mathrm{V}}$ \\
\hline$E_{\mathrm{V}}$ & Perovskite valence band maximum & $-5.4 \mathrm{eV}$ & Schulz et al. ${ }^{56}$ \\
\hline $\begin{array}{l}\hat{g}_{\mathrm{v}} \\
\hat{g}_{\mathrm{v}}\end{array}$ & Valence band density of states & $5.8 \times 10^{18} \mathrm{~cm}^{-3}$ & Brivio et al. ${ }^{59}$ \\
\hline$L_{\mathrm{d}}$ & Debye length & $1.5 \mathrm{~nm}$ & $=\left(\varepsilon_{\mathrm{p}} V_{\mathrm{T}} /\left(q N_{0}\right)\right)^{1 / 2}$ \\
\hline$N_{0}$ & $\mathrm{I}^{-}$volume-averaged vacancy density & $1.6 \times 10^{19} \mathrm{~cm}^{-3}$ & Walsh et al. ${ }^{46}$ \\
\hline$n(x=0)$ & Density of electrons in perovskite at $\mathrm{TiO}_{2}$ boundary & $4.8 \times 10^{14} \mathrm{~cm}^{-3}$ & Eqn (6) \\
\hline$n_{\mathrm{i}}$ & Intrinsic carrier density & $3.0 \times 10^{4} \mathrm{~cm}^{-3}$ & Eqn (8) \\
\hline$p(x=b)$ & Density of holes in perovskite at spiro boundary & $1.0 \times 10^{12} \mathrm{~cm}^{-3}$ & Eqn (7) \\
\hline$V_{\mathrm{T}}$ & Thermal voltage & $0.0257 \mathrm{~V}$ & Eqn (9) \\
\hline
\end{tabular}

Table 1 Parameters used in the drift-diffusion simulations. Reference numbers are given for those obtained from literature. The values given for the temperature-dependent quantities $L_{d}, n(x=0), n_{i}, p(x=b)$, and $V_{T}$ are for room temperature (298 K) 
and

$$
V_{\mathrm{T}}=\frac{k_{\mathrm{B}} T}{q},
$$

where $k_{\mathrm{B}}$ is the Boltzmann constant and $T$ the absolute temperature. Other symbols are defined in Table 1. According to Walsh et $a l^{46}$ the vacancy density $N_{0}$ is also temperature-dependent, although $N_{0}$ has been kept constant in this work due to the large uncertainty on the variability of this quantity.

In practice, since the simplified asymptotic model is solved only in the charge-free bulk region between the thin Debye layers, we require boundary conditions for the carrier densities at the edge of the Debye layers: $n\left(x=0^{+}\right)$for electrons and $p\left(x=b^{-}\right)$for holes. These conditions are obtained by assuming that within the Debye layers both electron and hole distributions are close to quasi-equilibrium and so are approximately Boltzmann distributed such that

$$
\begin{aligned}
& n(x) \approx n(x=0) \exp \left(+\frac{\phi(x)-\phi(0)}{V_{\mathrm{T}}}\right) \quad \text { very close to } x=0, \\
& p(x) \approx p(x=b) \exp \left(-\frac{\phi(x)-\phi(b)}{V_{\mathrm{T}}}\right) \quad \text { very close to } x=b .
\end{aligned}
$$

Given that there is a rapid jump in potential across the righthand Debye layer of size $\phi\left(b^{-}\right)-\phi(b)=\mathcal{V}_{+}$and across the lefthand Debye layer of size $\phi\left(0^{+}\right)-\phi(0)=\mathcal{V}_{-}$, the appropriate boundary conditions on the simplified asymptotic model are

$$
\begin{gathered}
n\left(x=0^{+}\right)=n(x=0) \exp \left(\frac{\mathcal{V}_{-}}{V_{\mathrm{T}}}\right), \\
p\left(x=b^{-}\right)=p(x=b) \exp \left(-\frac{\mathcal{V}_{+}}{V_{\mathrm{T}}}\right) .
\end{gathered}
$$

where $n(x=0)$ and $p(x=b)$ are given by (6) and (7).

Parameters found by fitting to dark current decay transient measurements are shown in Table 2. The room-temperature value of the $\mathrm{I}^{-}$vacancy diffusion coefficient $D_{+}$was tuned such that the time for the simulated dark current transient, as shown in Fig. 5, to reach a stable value matched the experiment. The electron and hole diffusion coefficients $\hat{D}_{\mathrm{n}}=\hat{D}_{\mathrm{p}}$ were adjusted to achieve the best possible agreement regarding the current transient behaviour observed prior to stabilisation at $T=298 \mathrm{~K}$, and were taken to be temperature independent. Savenije $e t$ al. ${ }^{60}$ find that the carrier diffusion coefficients are likely to vary as

Table 2 Parameter values derived from fitting the drift-diffusion simulations to experiment. $D_{+}$is temperature-dependent; the value at room temperature $(298 \mathrm{~K})$ is given here

\begin{tabular}{lll}
\hline Symbol & Description & Value \\
\hline$B$ & Bimolecular recombination factor & $4.1 \times 10^{-5} \mathrm{~cm}^{3} \mathrm{~s}^{-1}$ \\
$D_{+}$ & I $^{-}$vacancy diffusion coefficient & $7.5 \times 10^{-13} \mathrm{~cm}^{2} \mathrm{~s}^{-1}$ \\
$\hat{D}_{\mathrm{n}}$ & Electron diffusion coefficient & $0.21 \mathrm{~cm}^{2} \mathrm{~s}^{-1}$ \\
$\hat{D}_{\mathrm{p}}$ & Hole diffusion coefficient & $0.21 \mathrm{~cm}^{2} \mathrm{~s}^{-1}$ \\
$E_{\mathrm{A}}$ & I $^{-}$vacancy activation energy & $0.55 \mathrm{eV}$ \\
$V_{\mathrm{bi}}$ & Built-in voltage & $1.05 \mathrm{~V}$ \\
$v_{\mathrm{s}}$ & Surface recombination velocity & $0.058 \mathrm{~cm} \mathrm{~s}^{-1}$
\end{tabular}

$T^{-0.6}$ - this changes the values of $D_{+}$and $D_{-}$only very slightly over our temperature range, and negligibly compared to other parameters that vary exponentially with $T$. The need to fit $D_{+}, \hat{D}_{\mathrm{n}}$ and $\hat{D}_{\mathrm{p}}$ to our cell's experimental data arises from sensitivity of the diffusion coefficients to grain morphology, which varies from cell to cell.

According to Eames et al.,${ }^{47}$ the temperature dependence of $D_{+}$is given by

$$
D_{+}(T)=D_{\infty} \exp \left(-\frac{E_{\mathrm{A}}}{k_{\mathrm{B}} T}\right),
$$

where $E_{\mathrm{A}}$ is the activation energy for vacancy migration and $D_{\infty}$ is a constant determined by the frequency at which the $\mathrm{I}^{-}$ions attempt to move into a vacant site. Eames et al. $^{47}$ assume an attempt frequency of $10^{12} \mathrm{~Hz}$, resulting in the value of $D_{\infty}$ given in Table 1. Using the fitted parameter for $D_{+}$at $T=298 \mathrm{~K}$ in Table $2, E_{\mathrm{A}}$ is found to be $0.55 \mathrm{eV}$, marginally lower than the $E_{\mathrm{A}}=0.58 \mathrm{eV}$ found by Eames et $a l^{47}$

\subsection{Simulation protocol}

The asymptotic model was used to simulate the equilibration of the cell at $0 \mathrm{~V}$ applied potential in the dark for a time $t_{\text {eq }}$ before abruptly jumping to $V_{\text {jump, }}$, still in the dark, at $t=0$. During equilibration the areal charge density accumulates to $Q_{+}=-Q_{-}=Q_{\text {eq }}$ in the Debye layer adjacent to the spiro. For $T \geq 298 \mathrm{~K}, t_{\mathrm{eq}}=30 \mathrm{~s}$; for lower temperatures $t_{\mathrm{eq}}(T)$ is inversely proportional to the vacancy diffusion coefficient $D_{+}(T)$, which is given by (14). The initial condition is taken to be no net charge in the Debye layers $\left(Q_{+}=-Q_{-}=0\right)$ at $t=-t_{\text {eq }}$, although the procedure is not dependent on this. A second simulation is then performed, with $V=V_{\text {jump }}$ for $t>0$ and setting $Q_{+}=Q_{\mathrm{eq}}$ at $t=0$ as the initial condition. The second simulation yields the evolution of $Q_{+}$over time; this information can be used to find the current density $J(t)$ at any given point in time $t$.

\subsection{Cell fabrication and measurements}

For the experimental measurements, planar perovskite solar cells were made by spin coating of the mixed halide precursor solution of $\mathrm{CH}_{3} \mathrm{NH}_{3} \mathrm{I}$ and $\mathrm{PbCl}_{2}$ ( $3: 1$ molar ratio) in DMF, with a toluene-drip quenching step to facilitate fast crystallization of the film. The full fabrication method is explained in detail in Sakai et al. ${ }^{61}$ Compact $\mathrm{TiO}_{2}$ and spiro-OMeTAD were used as the electron and hole transport layers respectively. The active area of the cells was $0.19 \mathrm{~cm}^{2}$ as defined by the overlap of the FTO and gold contacts.

Current decay measurements were performed in the dark using a Solartron Modulab XM electrochemical system. Care was taken to ensure that all measurements were started from the same equilibrium condition by leaving the cell in the dark at open-circuit between measurements until the residual voltage dropped below $1 \mathrm{mV}$. This would take up to one hour at low temperatures. Temperature controlled measurements were performed using a Peltier element and DC temperature controller (Meerstetter Engineering).

We note that some MAPI cells fabricated using chloride precursors have been reported to contain residual chloride ions, and it is still an open question as to the effect of these ions 


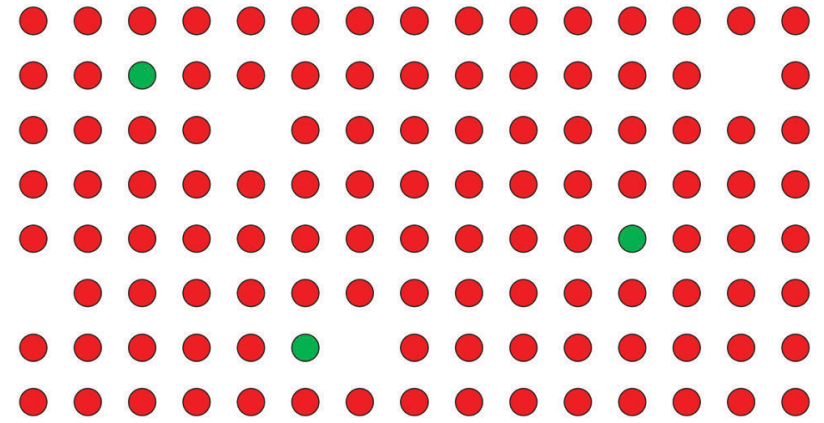

Fig. 3 Schematic of the arrangement of iodide ions (red), chloride ions (green) and vacancies in a generic lattice. The relative concentrations of chloride ions and halide vacancies compared to iodide ions are exaggerated.

(if any) on cell behaviour. Sakai et al. ${ }^{16}$ discuss the crystal growth in films produced using $\mathrm{PbCl}_{2}$. XRD shows that initially grains of $\mathrm{MAPbCl}_{3}$ form readily over MAPI grains. During the annealing phase, the XRD peak corresponding to $\mathrm{MAPbCl}_{3}$ disappears as the peak for MAPI grows. They note that, during crystal growth, the $\mathrm{MAPbCl}_{3}$ acts as a template for MAPI phases. During annealing, $\mathrm{I}^{-}$ions replace $\mathrm{Cl}^{-}$ions, forming apparently pure MAPI.

The source of controversy here is whether all of the chloride ions are actually removed from the film. In any case, there is a consensus that iodide ions are present in far greater numbers than chloride ions. Dar et al. ${ }^{62}$ used scanning transmission electron microscopy-energy dispersive spectroscopy (STEM-EDS) to find that films they produced using the chloride precursor route were 'chloride-free'. Yu et al. ${ }^{63}$ used energy dispersive X-ray spectrometry (EDX) and surface-sensitive X-ray photoelectron spectroscopy (XPS) and found what they called a 'negligible' amount of $\mathrm{Cl}$ in a fully annealed film. However, Yu et al. ${ }^{63}$ do note a weak XPS signal corresponding to $1 \% \mathrm{Cl}$ compared to I in the top $20 \mathrm{~nm}$ of their film. In addition, chloride and iodide ions attach to the same sites on the perovskite lattice. As halide migration in perovskites is vacancy mediated, this means that it is only necessary to account for one species of vacancy (see Fig. 3). Unless chloride ions are orders of magnitude more mobile than iodides, the values of vacancy diffusion coefficient and density will be determined solely by the values for iodide ions alone. Even in the case of extremely mobile chlorides the effect on the model is only to increase the effective vacancy mobility by a small amount. As MAPI cells that are not produced using chloride precursors exhibit the same hysteresis as ours, we are confident that it is iodide migration playing the major role in our cell and any chloride contamination does not significantly impact the results. We chose the chloride precursor method for this study as cells produced in this manner have been found to exhibit better photovoltaic performance. ${ }^{61}$

\section{Results}

Measured device current-voltage characteristics under illumination and the measurement statistics for the cell and batch from which it was chosen are given in the ESI.\$

\subsection{Ion motion}

The equilibrium conduction band profile across the perovskite layer is depicted in the upper panels of Fig. 4. Since the Debye layers in which the charge is located are narrow as described above, over $95 \%$ of the perovskite is free of net charge. Around equilibrium conditions the built-in voltage $V_{\mathrm{bi}}$ is fully screened by charge build-up in the Debye layers (in the form of $\mathrm{I}^{-}$ vacancies), so that the electric field $E_{\text {bulk }}$ in the net charge-free perovskite bulk is zero. The mobile positively charged vacancies pile up against the interface with the spiro, resulting in most of the charge $Q_{+}=Q_{\text {eq }}$ on that side of the perovskite film being concentrated within a Debye length $L_{\mathrm{d}}$ of the spiro interface. The negative charges on the other hand cannot move, so the charge $Q_{-}=-Q_{\text {eq }}$ on the $\mathrm{TiO}_{2}$ side is formed by the region being depleted of positively charged vacancies. While the fraction of iodide sites that are vacant in $\mathrm{CH}_{3} \mathrm{NH}_{3} \mathrm{PbI}_{3}$ is relatively large at around 1 in $750\left(0.4 \%\right.$ of unit cells have a vacancy), ${ }^{46}$ there are still far fewer sites vacant than there are occupied. This means there are only a small number of vacancies per unit volume that can be removed (filled) until there are none left, but many more vacancies can be piled up in the same volume. As a result, the charge $Q_{-}$on the $\mathrm{TiO}_{2}$ side accumulates over several $L_{\mathrm{d}}$. The greater extent of the Debye layer adjacent to the $\mathrm{TiO}_{2}$ compared to that adjacent to the spiro causes a greater magnitude of potential $\operatorname{drop}\left|\mathcal{V}_{-}\right|$across the former compared to the potential drop $\left|\mathcal{V}_{+}\right|$ across the latter. ${ }^{51}$ The value of $Q_{+}$after being held at $0 \mathrm{~V}$ for $30 \mathrm{~s}$, denoted $Q_{\text {eq }}$, was found to be $3.1 \mu \mathrm{C} \mathrm{cm}{ }^{-2}$.

The evolution of the conduction band profile for the first $8 \mathrm{~s}$ after jumping from the equilibrium condition to $V_{\text {jump }}=1 \mathrm{~V}$ is depicted in the lower panels of Fig. 4 . When the voltage is first switched from $0 \mathrm{~V}$ to $1 \mathrm{~V}, Q_{+}$is still equal to $Q_{\text {eq }}$, causing $E_{\text {bulk }}$ to be large and negative; specifically, $E_{\text {bulk }}=-V_{\text {jump }} / b$ at $t=0$. As time progresses, the positively charged iodide vacancies are driven by the negative $E_{\text {bulk }}$ to flow away from the spiro and back towards the $\mathrm{TiO}_{2}$, reducing $Q_{+},\left|Q_{-}\right|$and $\left|E_{\text {bulk }}\right|$. After $8 \mathrm{~s}$, $\left|E_{\text {bulk }}\right|$ has decayed to less than $5 \%$ of its initial value. The decay is non-exponential.

While these results are specifically for $\mathrm{TiO}_{2}$ :MAPI:spiro, we suggest the principles found here transfer over to other transport layer configurations as we can see no mechanism whereby the choice of transport layer could completely stop all ion motion. Other materials would likely alter the size of the layers or shift the timescale of ionic behaviour. The stages in different fabrication techniques for different layers and chemical effects between the layers and perovskite during and after fabrication may also play a role.

\subsection{Dark current decay transients}

Two dark current decay transient experiments were performed. In the first experiment, the absolute temperature $T$ was fixed at $298 \mathrm{~K}$ and different values of $V_{\text {jump }}$ were used. Fig. 5 shows the simulated (panel a) and measured (panel b) results of this experiment. The current density in this work is defined to be positive when generation exceeds recombination (i.e. power is being generated) and negative when recombination exceeds 
(a)
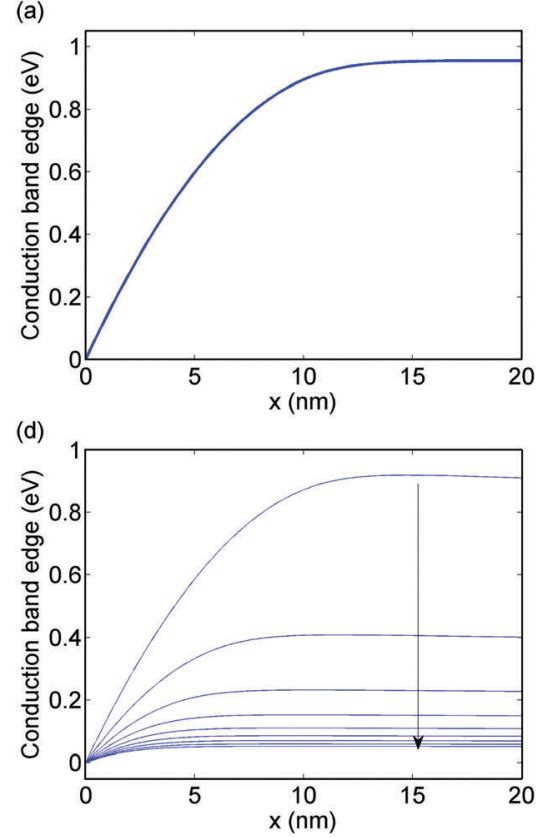

(b)

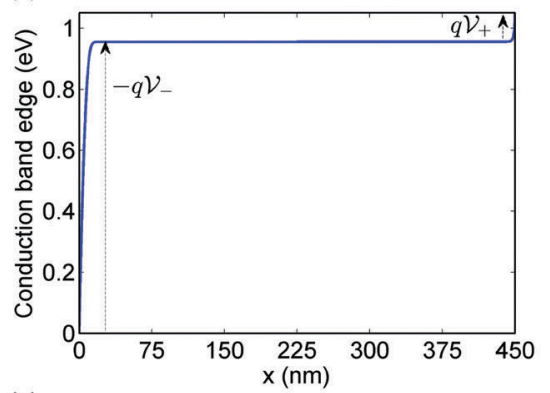

(e)

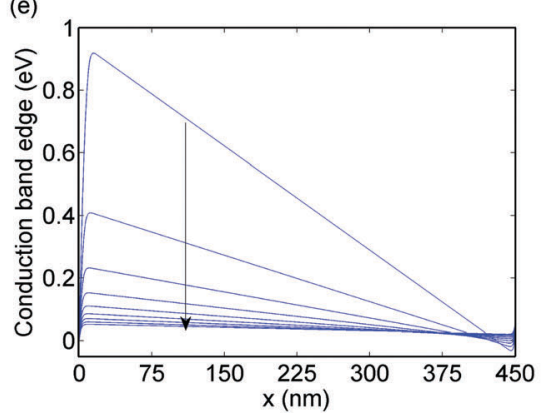

(c)
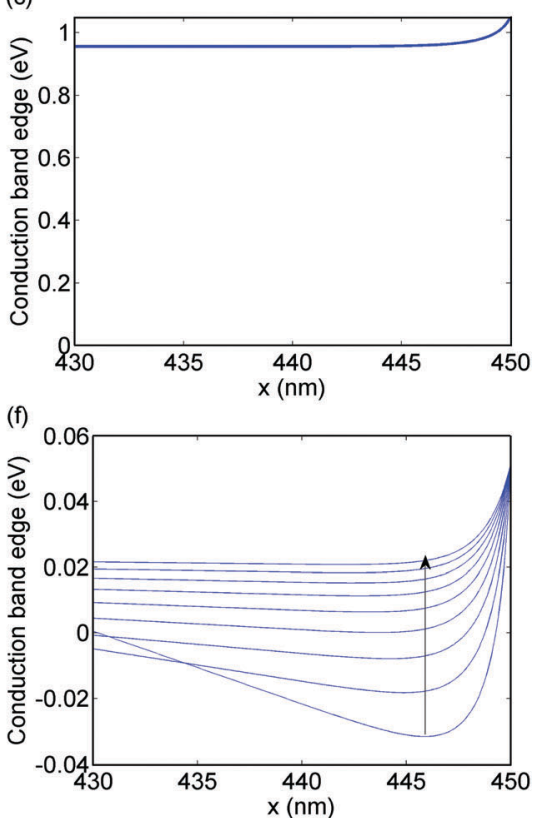

Fig. 4 Conduction band edge (CBE) across the $450 \mathrm{~nm}$ thick perovskite layer at $298 \mathrm{~K}$, relative to the CBE immediately next to the titania. The centre panels $b$ and $e$ show the entire perovskite layer. The left panels $a$ and $d$ show a close up of the Debye layer next to the titania; the right panels $c$ and $f$ show the Debye layer next to the spiro. Upper panels: CB profile after the cell has been held at $0 \mathrm{~V}$ for $30 \mathrm{~s}$, before the voltage jump. Lower panels: Evolution over time of the $\mathrm{CB}$ profile for the first $8 \mathrm{~s}$ after switching the applied voltage from $0 \mathrm{~V}$ to $V_{\text {jump }}=1 \mathrm{~V}$. The arrow indicates the direction of increasing time; the first line depicts the system immediately after the voltage jump at $t=0 \mathrm{~s}$, with successive lines at 1 second intervals.

generation (i.e. power is being consumed); it is therefore negative in the dark.

Assuming a diffusion coefficient $D_{+}=7.5 \times 10^{-13} \mathrm{~cm}^{2} \mathrm{~s}^{-1}$ for the $\mathrm{I}^{-}$vacancies, the agreement between simulation (Fig. 5a) and experiment (Fig. 5b) is good, both in terms of the magnitudes of the current densities and the timescale of the decay. Crucially, the current density maximum observed at $3 \mathrm{~s}$ for $V_{\text {jump }}=0.95 \mathrm{~V}$ in the measurement is predicted in the simulation - its origin is described below. However, the simulations fail to predict the minima observed for all $V_{\text {jump }}$ at around $1 \mathrm{~s}$, labelled minimum 1 on Fig. 5b, as well as the minima seen at around $20 \mathrm{~s}$ for $V_{\text {jump }} \geq 0.9 \mathrm{~V}$, labelled minimum 2 .

We believe the 'spike' at $t=0$ in the experiment is due to electron/hole displacement currents; these are beyond the scope of this work and provide no insight into ion motion. Minimum 1 occurs over short time scales on the order of $1 \mathrm{~s}$ and could have its origins in competing recombination pathways or motion of relatively fast $\mathrm{Li}^{+}$ions in the spiro hole transporting layer. Minimum 2 occurs at much longer timescales, on which the electric field $E_{\text {jump }}$ has been mostly compensated by the mobile ions. Possible causes of this second minimum include the very slow motion of methylammonium vacancies within the Debye layers, the creation of additional vacancies at grain boundaries in the perovskite film, or ions moving between the perovskite and electron/hole transporting layers (as alluded to by Richardson et $a l^{51}$ ). Further development of the model will be necessary to explain these discrepancies.

Fig. 6 shows the evolution of the bulk electric field $E_{\text {bulk, }}$, the areal charge density $Q_{+}$in the Debye layer adjacent to the spiro and the voltage drop $\mathcal{V}_{+}$across the same Debye layer. The bulk electric field $E_{\text {bulk }}$ is equal to $-V_{\text {jump }} / b$ at $t=0$ (when the jump from $0 \mathrm{~V}$ to $V_{\text {jump }}$ takes place) but returns to zero within $30 \mathrm{~s}$. The Debye charge $Q_{+}$decays from $Q_{\text {eq }}$ to a smaller stable value $Q_{\text {s }}$ over the same timescale. This decay occurs because vacancies move out of the layer on the spiro side of the perovskite into the bulk and from the bulk into the layer on the side with titania due to the reduced potential difference across the perovskite.

For values of $V_{\text {jump }}$ less than the built-in voltage, $0<Q_{\mathrm{s}}<Q_{\text {eq }}$, there is still a net potential difference across the device, though it is reduced to $V_{\mathrm{bi}}-V_{\text {jump }}$. As there is a smaller potential difference, charge flows out of the Debye layers into the bulk, reducing $Q_{+}$, $\left|Q_{-}\right|$and the widths of the layers to the extent needed to screen this smaller voltage. Lower values of $V_{\text {jump }}$ thus correspond to larger $Q_{\mathrm{s}}, \mathcal{V}_{+}$and $\left|\mathcal{V}_{-}\right|$, each in a nonlinear manner.

If $V_{\text {jump }}$ equals $V_{\mathrm{bi}}$ exactly, $Q_{\mathrm{s}}=0$. In this case there is no potential difference across the perovskite, and so nothing to keep the vacancies in the Debye layers. The Debye layers fully discharge such that the vacancy density is uniform across the entirety of the perovskite.

As discussed by Richardson et al. ${ }^{51}$ the quantities that have a major influence on recombination are $E_{\text {bulk }}, \mathcal{V}_{+}$and $\mathcal{V}_{-}$. Negative $E_{\text {bulk }}$ hinders charge extraction as charges in the bulk are pushed to the opposite side of the perovskite bulk to their respective transport layers, causing carriers to build up. The increase in carrier density enhances recombination, as in (4) and (5). After the initial jump in the applied potential $E_{\text {bulk }}$ jumps to a negative value, causing a sudden increase in recombination, before gradually relaxing to zero. 
(a)

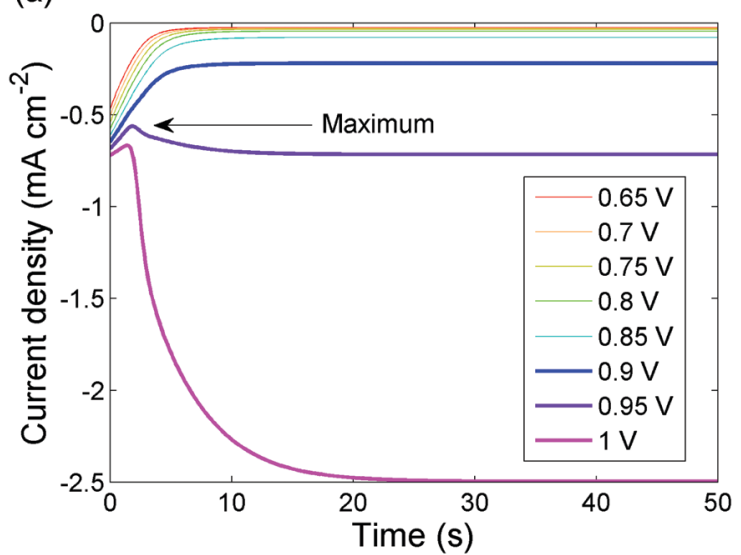

(b)

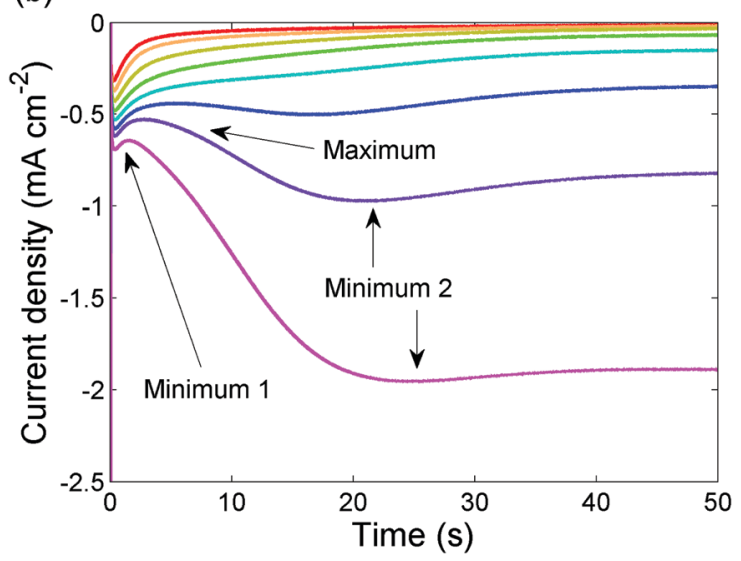

Fig. 5 (a) Simulated and (b) measured evolution of current density over $50 \mathrm{~s}$ following a jump from $0 \mathrm{~V}$ to $V_{\text {jump }}$ in the dark, for varying different values of $V_{\text {jump }}$ at room temperature $(298 \mathrm{~K})$. This gives good qualitative agreement between theory and experiment.

The effect of $\mathcal{V}_{+}$can be seen from the boundary condition (13). This equation demonstrates that a reduction in $Q_{+}$from its initial value $Q_{\text {eq }}$ reduces $\mathcal{V}_{+}$, and thereby increases $p\left(x=b^{-}\right)$at the edge of the spiro-side Debye layer and thus also the resulting recombination. The same logic applies for $\mathcal{V}_{-}$by considering the relation (12) between the electron density at the MAPI:TiO interface $n(x=0)$ and at the edge of the Debye layer on the titania side of the perovskite $n\left(x=0^{+}\right)$.

Fig. 7 shows the recombination pathways through the device. Due to the very deep valence band maximum of $\mathrm{TiO}_{2}$ and very shallow conduction band minimum of spiro, electrons must cross from the $\mathrm{CB}$ of the $\mathrm{TiO}_{2}$ to the $\mathrm{VB}$ of the spiro in order to form a complete circuit. This trajectory requires an electron in the conduction band to enter a hole in the valence band - in other words, recombination. A greater rate of recombination thus results in a more negative current as it is recombination which allows reverse current to traverse the energy landscape of a device. We identify three likely current pathways: (i) an electron in the titania $\mathrm{CB}$ may recombine across the interface with a hole in the MAPI VB, (ii) an electron in the MAPI CB may recombine with a hole in the MAPI VB and (iii) an electron in the MAPI CB may recombine across the interface with a hole in the spiro VB. (a)

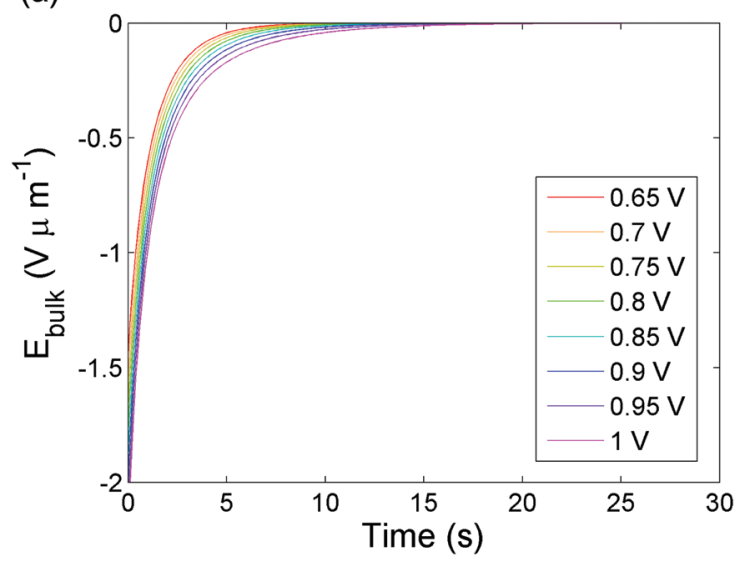

(b)

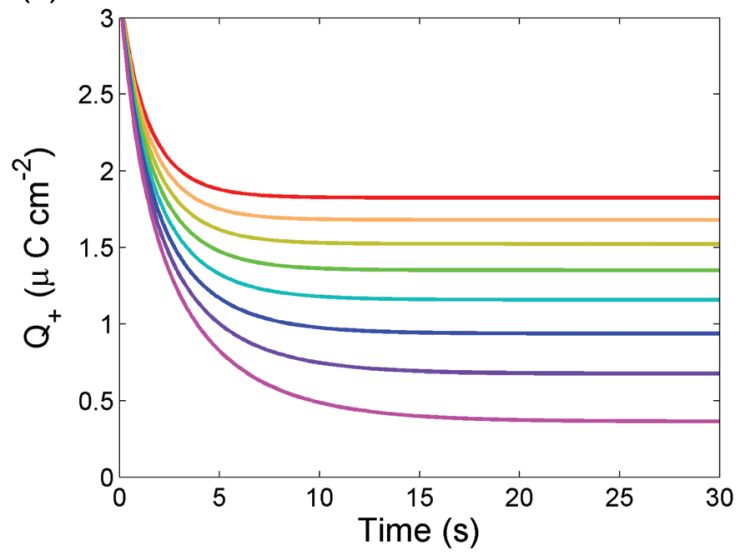

(c)

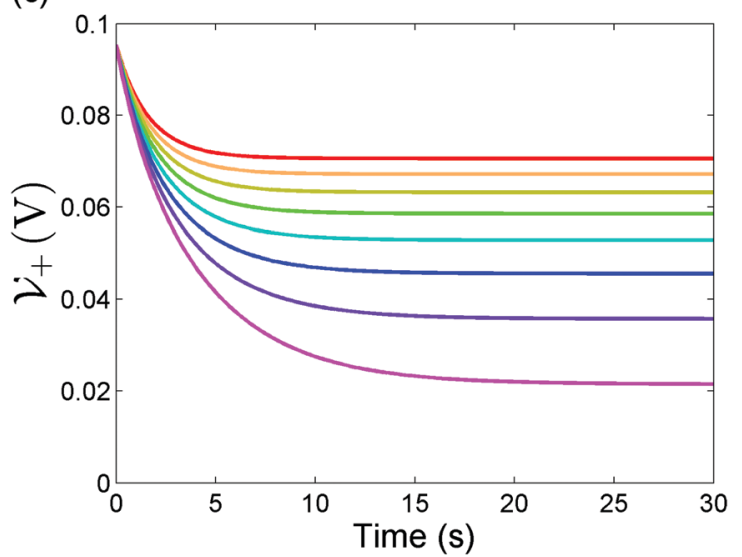

Fig. 6 Simulated (a) electric field $E_{\text {bulk }}$ between the Debye layers; (b) charge per unit area in the Debye layer next to the spiro; and (c) voltage drop $\mathcal{V}_{+}$across the same Debye layer.

Here, $\mathrm{TiO}_{2}$ :MAPI surface recombination (i) is modelled using the boundary condition (5) and bulk MAPI recombination (ii) using the bimolecular scheme (4). MAPI:spiro surface recombination (iii) is not modelled, as it was not found to have as significant an effect as (i).

It follows that a more negative $E_{\text {bulk }}$ results in a greater reverse current, while greater $\mathcal{V}_{+}$and $\left|\mathcal{V}_{-}\right|$result in less reverse current. As such, the decay of $E_{\text {bulk }}$ from negative to zero in Fig. 6a results in a decrease in magnitude of reverse current. 


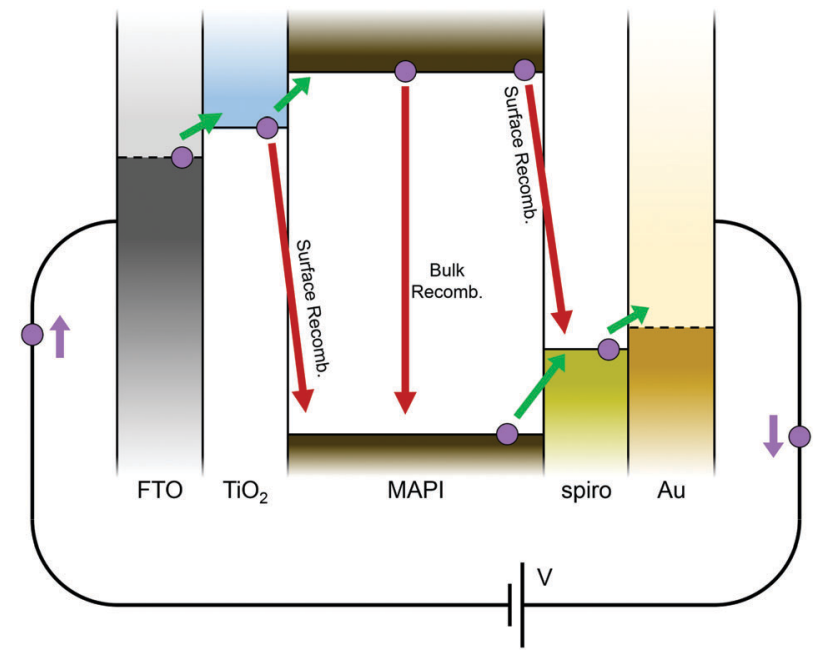

Fig. 7 Schematic of the possible paths reverse current can take through the device. The applied voltage $V$ 'pumps' electrons (purple circles) from the gold electrode to the FTO. Upward-pointing green arrows represent injection barriers between materials the electrons must overcome to increase in energy. Downward-pointing red arrows represent energetically favourable recombination pathways. Electron transport in the MAPI and spiro valence bands is hole mediated.

At the same time, the decay of $\mathcal{V}_{+}$from $\sim 0.1 \mathrm{~V}$ to a smaller value in Fig. 6c, and similar for $\mathcal{V}_{-}$, causes an increase in magnitude of reverse current.

The balance between these two effects explains the behaviour of the current density transient $J(t)$ in Fig. 5 . For $V_{\text {jump }} \leq 0.85 \mathrm{~V}$ the influence of $E_{\text {bulk }}$ dominates causing $J$ to increase then stabilise at a greater (less negative) value. For the case of $V_{\text {jump }} \geq 0.9$ in the experimental results, $E_{\mathrm{bulk}}$ dominates for a few seconds but then the $V$ dominate after, causing $J$ to increase to the maxima observed at $t$ of a few seconds, then decrease again before settling to a stable value. The simulated results show these maxima for $V_{\text {jump }} \geq 0.95 \mathrm{~V}$, but not $V_{\text {jump }}=0.9 \mathrm{~V}$. Notably, the maximum for $V_{\text {jump }}=1 \mathrm{~V}$ occurs at $t=1.5 \mathrm{~s}$ for both simulation and experiment.

\subsection{Temperature dependence}

A second dark current decay experiment was performed, where $V_{\text {jump }}$ was set to $1 \mathrm{~V}$ and the temperature was varied. Temperature variation was incorporated into the asymptotic model via eqn (6)-(14). For absolute temperatures $T<298 \mathrm{~K}$, holding the cell at $0 \mathrm{~V}$ for $30 \mathrm{~s}$ is not sufficient to reach equilibrium; the time $t_{\text {eq }}$ required to reach equilibrium is inversely proportional to the vacancy diffusion coefficient $D_{+}(T)$. Six values of absolute temperature $T$ were investigated and the results shown in Fig. 8.

As with the results in Fig. 6, neither of the current density minima observed in the measurements are present in the simulation results, although the agreement is otherwise good. In particular, the simulated and measured current densities decay on similar timescales for all the temperatures considered. If vacancy motion is a thermally activated mechanism (14), as proposed by Eames et al., ${ }^{47}$ this temperature dependence supports the hypothesis that the transients are a consequence of ion motion. (a)

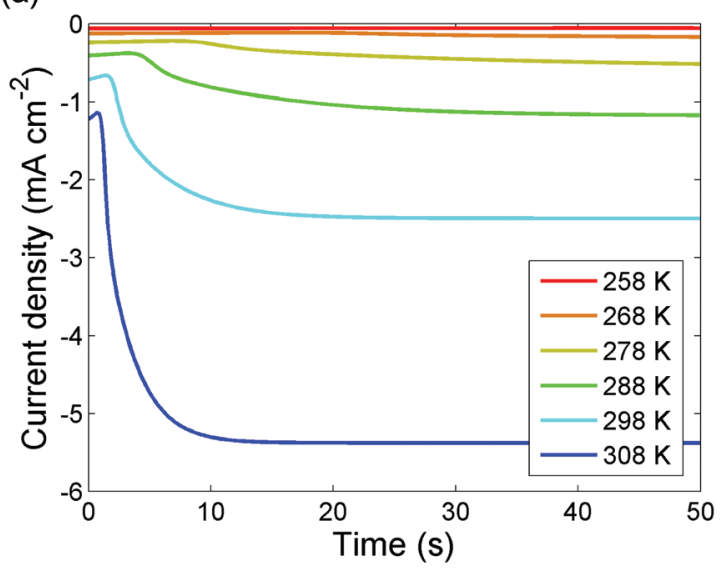

(b)

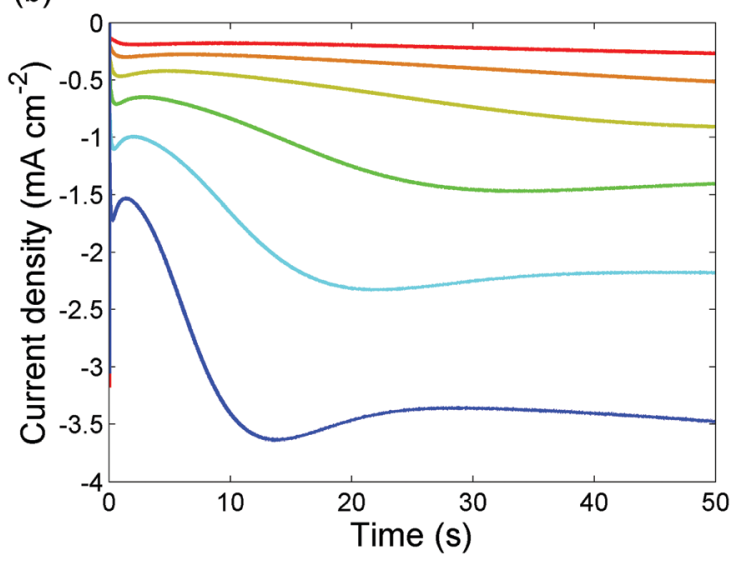

Fig. 8 Simulated (panels a) and measured (panel b) evolution of current density over $50 \mathrm{~s}$ following a jump from $0 \mathrm{~V}$ to $1 \mathrm{~V}$ in the dark, for six different values of absolute temperature $T$.

The large reduction in the saturation current density reached at large $t$ as the temperature is reduced is also predicted by the simulations. Notably saturation current density does not depend on ion motion; at long times the ion distribution reaches an equilibrium that is independent of temperature. Increases in temperature lead to increases in carrier concentrations on the perovskite boundaries (through (6) and (7)), hence to increased carrier concentrations throughout the perovskite layer and increased recombination. In turn, this increased recombination causes negative saturation currents of greater magnitude at high temperature (where carrier recombination is high) in comparison to low temperature (where carrier recombination is low). The fact that the experimental and theoretical saturation current densities do not match up is attributable to small errors in the Fermi levels used to calculate the boundary conditions (6) and (7) on the carrier concentration.

\section{Conclusions}

In conclusion, we have used dark current decay transient measurements to probe the physical behaviour of perovskite solar cells. This method has the significant advantage over other experimental procedures used to investigate charge transport in such cells that, 
by allowing the cell to equilibrate at open circuit in the dark prior to making a reading, the results are demonstrably reproducible. The results of these measurements have been explained in terms of the asymptotic drift-diffusion model of Richardson et al. ${ }^{51}$ and the comparison between theory and experiment has allowed us to verify the accuracy of this combined drift-diffusion model for $\mathrm{I}^{-}$vacancies, electrons and holes (which has already been used to explain current-voltage hysteresis loops) and gain further insight into the physics of perovskite solar cells. The ability to fit these transients over a wide range of temperatures, by postulating an Arrhenius dependence of the ion mobility on temperature (as in Eames et $a{ }^{4}{ }^{47}$ ), lends further support to the hypothesis that ion motion causes the transient behaviour. We close by remarking that this study has demonstrated that charge injection in perovskite solar cells (i.e. the current flow in the dark) is dependent on ion distribution.

\section{Author contributions}

SEJOK contributed to the design of the theoretical model, optimisation of its parameters, gathered simulation data and contributed to writing the manuscript. GR conceived of and formulated the model, designed methods for its numerical solution and contributed to writing the manuscript. AP measured the current transients. RGN measured JV curves (results did not make final version) and produced the TOC figure. JMC wrote the manuscript and contributed to interpretation of the model. GEE and NS fabricated the cells. HJS supervised and developed cell fabrication. JMF designed methods for numerical solution of the model using Chebfun. PJC supervised experimental measurements. ABW supervised theoretical work, contributed to the interpretation of the model and contributed to writing the manuscript.

\section{Acknowledgements}

The authors wish to acknowledge helpful discussions with Laurie Peter. The research leading to these results has received funding from the European Union Seventh Framework Programme FP7/2007-2013 under grant agreement 316494 and the UK Engineering and Physical Sciences Research Council grants $\mathrm{EP} / \mathrm{J} 017361 / 1$ \& EP/I01702X/1.

\section{References}

1 A. Kojima, K. Teshima, Y. Shirai and T. Miyasaka, J. Am. Chem. Soc., 2009, 131, 6050-6051.

2 T. C. Sum, S. Chen, G. Xing, X. Liu and B. Wu, Nanotechnology, 2015, 26, 342001.

3 S. D. Stranks and H. J. Snaith, Nat. Nanotechnol., 2015, 10, 391-402.

4 G. Niu, X. Guo and L. Wang, J. Mater. Chem. A, 2015, 3, 8970-8980.

5 T. Miyasaka, Chem. Lett., 2015, 44, 720.

6 N.-G. Park, J. Phys. Chem. Lett., 2013, 4, 2423-2429.
7 Y. Zhang, M. Liu, G. E. Eperon, T. C. Leijtens, D. McMeekin, M. Saliba, W. Zhang, M. de Bastiani, A. Petrozza, L. M. Herz, M. B. Johnston, H. Lin and H. J. Snaith, Mater. Horiz., 2015, 2, 315-322.

8 A. Dualeh, T. Moehl, N. Tétreault, J. Teuscher, P. Gao, M. K. Nazeeruddin and M. Grätzel, ACS Nano, 2014, 8, 362-373.

9 H. J. Snaith, A. Abate, J. M. Ball, G. E. Eperon, T. Leijtens, N. K. Noel, S. D. Stranks, J. T.-W. Wang, K. Wojciechowski and W. Zhang, J. Phys. Chem. Lett., 2014, 5, 1511-1515.

10 R. S. Sanchez, V. Gonzalez-Pedro, J.-W. Lee, N.-G. Park, Y. S. Kang, I. Mora-Sero and J. Bisquert, J. Phys. Chem. Lett., 2014, 5, 2357-2363.

11 E. L. Unger, E. T. Hoke, C. D. Bailie, W. H. Nguyen, A. R. Bowring, T. Heumuller, M. G. Christoforo and M. D. McGehee, Energy Environ. Sci., 2014, 7, 3690-3698.

12 W. Tress, N. Marinova, T. Moehl, S. M. Zakeeruddin, M. K. Nazeeruddin and M. Grätzel, Energy Environ. Sci., 2015, 8, 995-1004.

13 C. C. Stoumpos, C. D. Malliakas and M. G. Kanatzidis, Inorg. Chem., 2013, 52, 9019-9038.

14 L. M. Herz, Annu. Rev. Phys. Chem., 2016, 67, 65-89.

15 T. J. Jacobsson, J.-P. Correa-Baena, M. Pazoki, M. Saliba, K. Schenk, M. Grätzel and A. Hagfeldt, Energy Environ. Sci., 2016, 9, 1706-1724.

16 G. E. Eperon, S. D. Stranks, C. Menelaou, M. B. Johnston, L. M. Herz and H. J. Snaith, Energy Environ. Sci., 2014, 7, 982-988.

17 G. E. Eperon, R. J. S. Giuseppe, M. Paternò, A. A. H. Andrea Zampetti, F. Cacialli and H. J. Snaith, J. Mater. Chem. A, 2015, 3, 19688-19695.

18 M. Saliba, T. Matsui, J.-Y. Seo, K. Domanski, J.-P. CorreaBaena, M. K. Nazeeruddin, S. M. Zakeeruddin, W. Tress, A. Abate, A. Hagfeldt and M. Grätzel, Energy Environ. Sci., 2016, 9, 1989-1997.

19 N. Pellet, P. Gao, G. Gregori, T.-Y. Yang, M. K. Nazeeruddin, J. Maier and M. Grätzel, Angew. Chem., Int. Ed., 2014, 53, 3151-3157.

20 N. K. Noel, S. D. Stranks, A. Abate, C. Wehrenfennig, S. Guarnera, A.-A. Haghighirad, A. Sadhanala, G. E. Eperon, S. K. Pathak, M. B. Johnston, A. Petrozza, L. M. Herz and H. J. Snaith, Energy Environ. Sci., 2014, 7, 3061-3068.

21 S. Colella, E. Mosconi, P. Fedeli, A. Listorti, F. Gazza, F. Orlandi, P. Ferro, T. Besagni, A. Rizzo, G. Calestani, G. Gigli, F. D. Angelis and R. Mosca, Chem. Mater., 2013, 25, 4613-4618.

22 K. Cao, H. Li, S. Liu, J. Cui, Y. Shen and M. Wang, Nanoscale, 2016, 8, 8839-8846.

23 X. Li, D. Bi, C. Yi, J.-D. Décoppet, J. Luo, S. M. Zakeeruddin, A. Hagfeldt and M. Grätzel, Science, 2016, 353, 58-62.

24 D. P. McMeekin, G. Sadoughi, W. Rehman, G. E. Eperon, M. Saliba, M. T. Hörantner, A. Haghighirad, N. Sakai, L. Korte, B. Rech, M. B. Johnston, L. M. Herz and H. J. Snaith, Science, 2016, 351, 151-155.

25 Q. Tai, P. You, H. Sang, Z. Liu, C. Hu, H. L. W. Chan and F. Yan, Nat. Commun., 2016, 7, 11105. 
26 M. M. Lee, J. Teuscher, T. Miyasaka, T. N. Murakami and H. J. Snaith, Science, 2012, 338, 643-647.

27 Y. Shao, Z. Xiao, C. Bi, Y. Yuan and J. Huang, Nat. Commun., 2014, 5, 5784.

28 N. Tripathi, M. Yanagida, Y. Shirai, T. Masuda, L. Han and K. Miyano, J. Mater. Chem. A, 2015, 3, 12081.

29 Y. Hou, C. O. R. Quiroz, S. Scheiner, W. Chen, T. Stubhan, A. Hirsch, M. Halik and C. J. Brabec, Adv. Energy Mater., 2015, 5, 1501056.

30 H. Yoon, S. M. Kang, J.-K. Lee and M. Choi, Energy Environ. Sci., 2016, 9, 2262.

31 A. T. Mallajosyula, K. Fernando, S. Bhatt, A. Singh, B. W. Alphenaar, J.-C. Blancon, W. Nie, G. Gupta and A. D. Mohite, Mater. Today, 2016, 3, 96-102.

32 L. Cojocaru, S. Uchida, P. V. V. Jayaweera, S. Kaneko, J. Nakazaki, T. Kubo and H. Segawa, Chem. Lett., 2015, 44, 1750-1752.

33 D. Bryant, S. Wheeler, B. C. O'Regan, T. Watson, P. R. F. Barnes, D. Worsley and J. Durrant, J. Phys. Chem. Lett., 2015, 6, 3190-3194.

34 H.-S. Kim, I.-H. Jang, N. Ahn, M. Choi, A. Guerrero, J. Bisquert and N.-G. Park, J. Phys. Chem. Lett., 2015, 6, 4633-4639.

35 E. Guillén, F. J. Ramos, J. A. Anta and S. Ahmad, J. Phys. Chem. C, 2014, 118, 22913-22922.

36 A. Pockett, G. E. Eperon, T. Peltola, H. J. Snaith, A. Walker, L. M. Peter and P. J. Cameron, J. Phys. Chem. C, 2015, 119, 3456-3465.

37 A. Baumann, K. Tvingstedt, M. C. Heiber, S. Väth, C. Momblona, H. J. Bolink and V. Dyakonov, APL Mater., 2014, 2, 081501.

38 B. C. O'Regan, P. R. F. Barnes, X. Li, C. Law, E. Palomares and J. M. Marin-Beloqui, J. Am. Chem. Soc., 2015, 137, 5087-5099.

39 J. M. Frost, K. T. Butler, F. Brivio, C. H. Hendon, M. van Schilfgaarde and A. Walsh, Nano Lett., 2014, 14, 2584-2590.

40 J. Beilsten-Edmands, G. E. Eperon, R. D. Johnson, H. J. Snaith and P. G. Radaelli, Appl. Phys. Lett., 2015, 106, 173502.

41 H.-S. Duan, H. Zhou, Q. Chen, P. Sun, S. Luo, T.-B. Song, B. Bob and Y. Yang, Phys. Chem. Chem. Phys., 2015, 17, 112-116.

42 J. H. Heo, D. H. Song, H. J. Han, S. Y. Kim, J. H. Kim, D. Kim, H. W. Shin, T. K. Ahn, C. Wolf, T.-W. Lee and S. H. Im, Adv. Mater., 2015, 27, 3424-3430.

43 O. Almora, I. Zarazua, I. Mas-Marza, E. Mora-Sero, J. Bisquert and G. Garcia-Belmonte, J. Phys. Chem. Lett., 2015, 6, 1645-1652.

44 J. Xu, A. Buin, A. H. Ip, W. Li, O. Voznyy, R. Comin, M. Yuan, S. Jeon, Z. Ning, J. J. McDowell, P. Kanjanaboos, J.-P. Sun,
X. Lan, L. N. Quan, D. H. Kim, I. G. Hill, P. Maksymovych and E. H. Sargent, Nat. Commun., 2015, 6, 7081.

45 T.-Y. Yang, G. Gregori, N. Pellet, M. Grätzel and J. Maier, Angew. Chem., Int. Ed., 2015, 54, 7905.

46 A. Walsh, D. O. Scanlon, S. Chen, X. G. Gong and S.-H. Wei, Angew. Chem., Int. Ed., 2015, 127, 1811.

47 C. Eames, J. M. Frost, P. R. F. Barnes, B. C. O'Regan, A. Walsh and M. S. Islam, Nat. Commun., 2015, 6, 7497.

48 J. M. Azpiroz, E. Mosconi, J. Bisquert and F. de Angelis, Energy Environ. Sci., 2015, 8, 2118-2127.

49 J. Haruyama, K. Sodeyama, L. Han and Y. Tateyama, J. Am. Chem. Soc., 2015, 137, 10048-10051.

50 S. Meloni, T. Moehl, W. Tress, M. Franckevičius, M. Saliba, Y. H. Lee, P. Gao, M. K. Nazeeruddin, S. M. Zakeeruddin, U. Rothlisberger and M. Grätzel, Nat. Commun., 2016, 7, 10334.

51 G. Richardson, S. E. J. OKane, R. G. Niemann, T. A. Peltola, J. M. Foster, P. J. Cameron and A. B. Walker, Energy Environ. Sci., 2016, 9, 1476-1485.

52 C. Li, S. Tscheuschner, F. Paulus, P. E. Hopkinson, J. Kießling, A. Köhler, Y. Vaynzof and S. Huettner, Adv. Mater., 2016, 28, 2446-2454.

53 Y. Zhao, C. Liang, H. Zhang, D. Lu, D. Tian, G. Li, X. Jing, W. Zhang, W. Xiao, Q. Liu, F. Zhang and Z. He, Energy Environ. Sci., 2015, 8, 1256-1260.

54 S. van Reenen, M. Kemerink and H. J. Snaith, J. Phys. Chem. Lett., 2015, 6, 3808-3814.

55 R. Gottesman, L. Gouda, B. S. Kalanoor, E. Haltzi, S. Tirosh, E. Rosh-Hodesh, Y. Tischler, A. Zaban, C. Quarti and E. Mosconi, et al., J. Phys. Chem. Lett., 2015, 6, 2332-2338.

56 P. Schulz, E. Edri, S. Kirmayer, G. Hodes, D. Cahen and

A. Kahn, Energy Environ. Sci., 2014, 7, 1377-1381.

57 G. Richardson, Math. Med. Biol., 2009, 26, 201-224.

58 P. J. Cameron and L. M. Peter, J. Phys. Chem. B, 2003, 107, 14394-14400.

59 F. Brivio, K. T. Butler, A. Walsh and M. van Schilfgaarde, Phys. Rev. B: Condens. Matter Mater. Phys., 2014, 89, 155204.

60 T. J. Savenije, C. S. Ponseca, L. Kunneman, M. Abdellah, K. Zheng, Y. Tian, Q. Zhu, S. E. Canton, I. G. Scheblykin, T. Pullerits, A. Yartsev and V. Sundström, J. Phys. Chem. Lett., 2014, 5, 2189-2194.

61 N. Sakai, S. Pathak, H.-W. Chen, A. A. Haghighirad, S. D. Stranks, T. Miyasaka and H. J. Snaith, J. Mater. Chem. A, 2016, 4, 4464-4471.

62 M. I. Dar, N. Arora, P. Gao, S. Ahmad, M. Grätzel and M. K. Nazeeruddin, Nano Lett., 2014, 14, 6991-6996.

63 H. Yu, F. Wang, F. Xie, W. Li, J. Chen and N. Zhao, Adv. Funct. Mater., 2014, 24, 7102-7108. 